\title{
NOTE TECHNIQUE. PROTECTION DU BOIS DES RUCHES. LE PARAFFINAGE.
}

\author{
l'AR \\ J. FRESNAYE \\ Station expérimentale d'Apiculture, Montfavet. \\ I ${ }^{\mathrm{r}}$ PAR'TIE.

\section{I. - INTRODUCTION}

I'Apiculteur a longtemps logé ses abeilles dans des ruches de paille, des troncs d'arbres creux, cles paniers d'osier on mêne des récipients de terre cuite. Il ne se préoccupait pas alors d'une protection efficace et durable de l'habitat de ses colonies: il se contentait du dôme de paille étalé en parapluie et sur lecpuel l'eau glisse sans pénétrer.

1) furis la naissance de l'apiculture moderne utilisant la ruche en bois, a cadres mobiles, le problème de la protection des bois s'est posé avec acuité sans être jamais résolu. Aucun des revêtements employés jusqu'à présent n'a donné entière satisfaction. Il semble d'ailleurs que beaucoup d'apiculteurs 11'emploient (qu'un petit nombre de procédés et ignorent les très grands progrès techniques en matière de protection du bois. C'est pourquoi la Station expérimentale d'Apiculture de Montfavet a entrepris des expériences dans le but de déterminer les meilleurs revêtements pour les ruches modernes.

\section{II. - LES DONNÉES DU PROBLEME DE LA PROTECTION DES RUCHES}

L'extrême variété des procédés utilisés jusqu'à ce jour pour la protection des bois en ceuvre à l'extérieur prouve la complexité du problème, complexité qui tient à la différence des types de protections recherchés. Selon les produits, l'action se manifeste surtout à l'égard des insectes, des champignons ou des défornuations.

Nous citerons parmi les revêtements utilisables.

Io L'huile de lin.

$2^{\circ}$ Les peintures à $1^{\prime}$ huile de lin.

$3^{\circ}$ Les peintures bitumineuses. 
$4^{\circ}$ Les peintures et vernis aux résines artificielles.

$5^{\circ}$ Les dérivés du caoutchouc.

$6^{\circ}$ Les produits hydrofuges.

$7^{\circ}$ I es fongicides et insecticides.

$S^{\circ} \mathrm{L}$ a paraffine.

$9^{\circ}$ Les résines servant à l'imprégnation par diffusion osmotique.

I $0^{\circ}$ Certains produits utilisés dans les imprégnations en vase clos.

I e choix entre ces différents procédés de préservation se trouve compliqué par des contingences d'ordre purement apicole. La difficulté jusqu'alors insurmontée est d'obtenir une protection durable et polyvalente nais d'un prix de revient peu élevé.

Iixaminons tout d'abord les qualités que devrait posséder le revêtement idéal pour la protection du bois des ruches soumis aux agents naturels.

I ${ }^{\circ}$ 'adhérence au support doit être parfaite. Ia plus grande attention doit être accordée à l'adaptation de la cotiche d'impression au bois ainsi qu'à sa compatibilité avec les couches de finition.

$2^{\circ}$ La souplesse du film protecteur est très importante car le bois est hygroscopique, donc sujet à des variations dimensionnelles importantes entraînant des craquelures dans les revêtements qui ne pourraient se prêter à ces variations.

$3^{\circ}$ Insecticide et fongicide. Le bois peut servir de lemeure et de nouriture à certains insectes comme les Tormites, les Capricornes, les Lyctus, les Anobium. Des champignons s'y développent également, causant des pourritures diverses. I e revêtement devra opposer un obstacle à tous par sa clureté ou mieux par sa toxicité.

$4^{\circ}$ Durabilité. S'il faut appliquer à nouveau un produit tous les deux ou trois ans on ne peut le considérer comme durable. Cette restriction s'applique à certains produits employés actuellement et en particulier at1x peintures à l'huile.

$5^{\circ}$ Facilité d'application. L'application doit être à la portée des apiculteurs. L'apiculture française est surtout artisanale. Ceux qui la pratiquent ne disposent donc pas d'appareils de type industriels. Cela élimine (pour l'instant) les imprégnations dites en vase clos, procédé dont nous parlerons plus loin.

$6^{\circ}$ Economie. Les apiculteurs professionnels ont en moyenne de cinc cents à mille ruches à protéger. Én ajoutant les hausses cela représente une surface de bois assez importante. Le produit doit être d'un prix de revient abordable.

$7^{\circ}$ Non toxicité pour les abeilles. Une ruche imprégnée de carbonyle par exemple, ne peut être utilisée que trois ou quatre mois après application, les vapeurs étant répulsives et toxiques pour les abeilles. Pour la même raison certains produits, de fabrication récente pour la plupart, ne peuvent être enployés en apiculture, tout au moins avant de sévères contrôles.

so Protection contre les radiations solaires. Cette protection peut être assurée grâce à l'emploi de teintes claires qui assurent une bonne réflexion du rayonnement.

$9^{\circ}$ Perméabilité faible. La perméabilité plus on moins problénatique des ruches est l'objet de maintes superstitions apicoles. Il convient donc d'examiner cette question un peu plus à fond que les précédentes.

Certains apiculteurs placés dans des régions moins favorisées que le Midi de la France donnent leur préférence aux ruches sans revêtement ni protection 
d'aucune sorte. 1)'autres enduisent seulement les assemblages, bien que cela soit d'une esthétique discutable. Cela leur permet, disent-ils, en laissant le bois perméable de lutter contre la condensation excessive à l'intérieur des ruches pendant l'hiver, condensation cui va jusqu'au ruissellement le long des parois et à la formation de givre par tempss froid. On sait en effet que les abeilles ne maintiennent la température nécessaire à leur survie qu'à l'intérieur de la grappe et non pas dans les zones immédiatement adjacentes (I AviE, I 954$)(\mathbf{r})$.

Or il semble bien yuc la perméabilité du bois n'entre pas en jeu clans le phénomène ou n'y cntre que pour une part très faible. Fin effet Bijıni, (2) a revêtu entièrement une ruche d'une pellicule de cantchonc et les abeilles n'ont pas été plus incommodées que celles des ruches voisines non soumises au même traitement. I)'ailleurs la perméabilité de planches anssi épaisses que celles des ruches est minine. Restent les propriétés du bois en tant que iolant hygrométriqut', l'eau pénétrant dans les pores du hois aux périodes humides pour être restituée à l'extérieur par temps sec. $\mathrm{IABI}_{\mathrm{A}}\left(\mathrm{KOV}^{\circ}\right.$ (3) a bien mis en évidence cette propriété et son importance dans la biologie des coléoptères xylophages. Mais ceci ne joue (lu'à la surface du bois et l'épaisseur de la paroi n'entre pas en ligne de compte dès lors qu'elle détrasse plusieurs centimètres.

1)'autre part, les aboilles bouchent les moindres lentes avec do la propolis et en enduisent mêne les parois. Ia conclusion, surprenante, à lacuelle nous nous voyons contraints est que la ruche devient en quelque sorte étanche de l'intérieur en restant perméable dans les conches plus externes.

Nous estimons donc en définitive que l'on doit rechercher avant tout l'inperméabilité afin de climinuer le plus possible les échanges d'humidité. clonc les variations dimensionnelles du bois qui provoquent une "fatigue " du filn de peinture et diminuent sa tenue et sa conservation.

\section{III. - LES SOLUTIONS ACTUELLES}

\section{1) Procédís couramment utilisés actuellement.}

Connaissant les qualités requises d'un bon revêtement, il devient possib'e d'établir la valeur relative des différents produits actuellement utilisés. I'armi les plus employés nous retiendrons :

-.. le carbonyle,

le carbonyle arec adjonction d'huile cle lin,

-... le carbonyle reconvert de paraffine,

la peinture à l'huile de lin,

--- la peinture à l'huile de lin et pignent d'aluminium,

-.. - la peinture glycérophtalique,

la paraffine.

Les méthodes d'imprégnations utilisées par les apiculteurs sont:

--- le badigeonnage an pincean,

- le pistolet à air comprimé,

-.. le trempage à froid,

-- le trempage à chand.

I.e tableau ci-joint permettra de comparer clairement les qualités et défauts cles différents revêtements en cause et les procédés d'application correspondant. I,es prix de revient sont indiqués pour la matière première seule à l'exclu- 


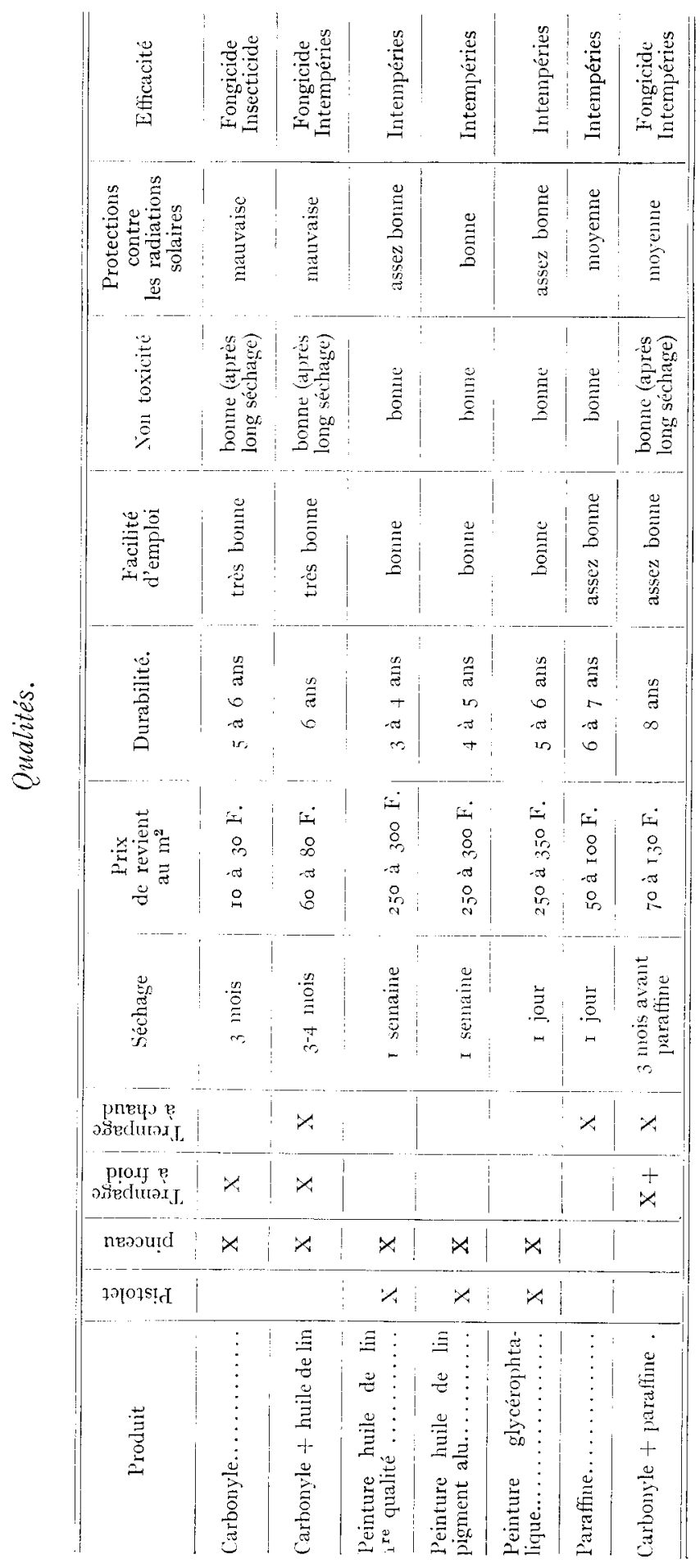


sion de la main-d'ouvre. Ils sont indiqués par ruche, sans hausse (il s'agit de ruches Dadant Io cadres; ce modèle, très employé en France, correspond à peu près à une surface de un mètre carré de bois à préserver). Les prix sont susceptibles de variations assez importantes en fonction des conditions d'achat des revêtements (achat en gros, achat à l'usine supprimant les intermédiaires) et aussi en fonction des utilisateurs eux-mêmes.

Dressons maintenant un inventaire détaillé de tous les revêtements utilisables sur bois. Certains sont employés en apiculture, d'autres seraient susceptibles de l'être, d'autres encore ne peuvent l'être et ne seront cités clu'à titre indicatif.

\section{Revêtements utilisables à l'échelon artisanal.}

Ce sont les revêtements dont l'application n'implique pas l'intervention d'appareillage industriel.

Io L'huile de lin. - I'huile de lin est la plus utilisée en peinture. On distingue

Huile de pays : très colorée; de caractéristiques variables suivant le mode d'extraction et le temps de stockage.

Huile de la Baltique : de qualité variable et de degré d'acidité élevé.

Huile Plata : également acide, convient pour la fabrication des peintures courantes.

Huile Bombay : pâle, peu acide, c'est la meilleure qualité connue sur le marché.

Les huiles de lin sont transformées par la chaleur, et acquièrent des propriétés particulières qui les font rechercher de préférence aux huiles crues.

Les différentes cuissons donnent :

Huiles cuites : elles sont chauffées à $150^{\circ}$ en présence de siccatifs.

Huiles soufflées : elles sont sounises à un courant d'air au cours de la cuisson à $I 80^{\circ}$. Elles s'oxydent et sont plus siccatives.

Standolies: Huiles ctuites à $300^{\circ}$ en atmosphère inerte. Les peintures fabriquées avec des standolies sont plus résistantes aux intempéries.

L'huile de lin est couramment employée non pigmentée en menuiserie extérieure. En apiculture les utilisateurs enduisent en général leurs ruches au carbonyle quelques mois avant d'appliquer l'huile de lin, ou font un mélange des deux produits avant l'application. Le prix de revient est peu élevé, trente à cinquante francs au mètre carré. La préservation est de courte durée, deux ans environ, et la protection contre les radiations solaires nulle. L'huile de lin appliquée à chaud $\left(50^{\circ}-60^{\circ}\right)$ assurerait wne protection bien meilleure.

L'huile de bois de chine et l'hwile d'abrazin proviennent d'espèces végétales voisines. Elles sont plus siccatives que l'huile de lin et sont rarement employées crues. Les standolies de ces huiles confèrent aux peintures une grande rapidité de séchage, une plus grande résistance aux intempéries ainsi qu'une imperméabilité supérieure à celle de l'huile de lin. Leur prix de revient en limite l'emploi et on les utilise comme adjuvant dans les peintures à l'huile de lin.

L'huile de ricin, l'huile de pépin de raisin, l'huile de pavot, l'huile de soja sont peu employées pas plus que bien d'autres qu'il est inutile de citer. 


\section{Peinture à l'huile.}

La protection cles bois en cuvre par cles peintures à bénéficié jusqu'à présent de la faveur générale. I.es peintures à l'huile de lin se composent :

-. de liant (huile de lin, huile de bois de chine),

- de pigments (blanc de zinc, pigments divers),

-.-- de solvants (essence de térébenthine, White spirit),

- de charges (dolonies, talc, sulfate de baryum).

Ces peintures qui furent sans concurrence pendant longtemps sont actuellennent dépassées par les revêtements à base de produits synthétiques : leur emploi, notamment à l'extérieur, tombe en désuétude surtout en raison de leur courte durée cle préservation. L,e clonliage, l'écaillage, causés par une mauvaise adhérence au substrat et une faible résistance aux intempéries en sont les défauts majeurs. Ces défauts sont atténués dans les peintures cle très bonne qualité préparées exclusivenent avec de l'huile de lin cuite, où le pourcentage d'huile dépasse 30 1\% roo. I a peinture est alors brillante et lisse, l'attaque des agents de corrosion est plus faible.

Les pigments métalliques peuvent être utilisés pour obtenir un effet décoratif mais dans les peintures d'extérieur leur principale qualité est d'augmenter la résistance du film aux intempéries. Remontant à la surface de la couche au cours du séchage, ils forment un écran continu très résistant qui augmente la durabilité du revêtement. Le principal pigment métallique employé est l'aluminium. Il est olstenu par broyage de feuilles ou de déchets d'aluminium en atmosphère inerte afin d'éviter l'oxydation. I a poussière, obtenue extrêmement fine, est enrobée de stéarine pour éviter l'oxydation et l'agglomération de grains.

Les charges sont employées pour des raisons techniques et économiques. Lilles se présentent en général sous la forme de poudres blanches. I,eur pouvoir pismentaire est faible. Hmployées en petite yuantité, moins de 7 p. Ioo, elles ont un rôle stabilisateur en favorisant la suspension des pigments dans le liant. In trop important pourcentage de charge diminue la qualité d'une peinture. L'oxyde de titane, pigment dont le pouvoir couvrant est considérable se prête particulièrement bien à l'adjonction de clıarge.

\section{Peintures et vernis bitumineux.}

Les peintures et vernis bitumineux sont constitués par des bitumes naturels ou par des brais de pétrole ou de houille, dissous dans des solvants benzéniques. I es vernis sont noirs et sont utilisés dans le bâtiment pour les ferrures, et en fumisterie. Ires peintures sont en général pignentées par de la poudre ('aluminium et sont utilisées comme antirouille sous la dénomination de "braialuminium " (4) S Sur bois à l'extérieur le prix de revient est inférieur à celui des peintures à l'huile, mais les qualités sont égalenent moins honnes: la plupart le ces peintures s'écaillent rapidenent.

\section{Peintures aux résines artificielles.}

I,es peintures aux résines artificielles sont nombreuses. Il suffira de connaitre les composants, le mode d'application, les qualités de préservation et de durabilité des différentes peintures sỵthétiques mises à notre disposition par l'industrie. 


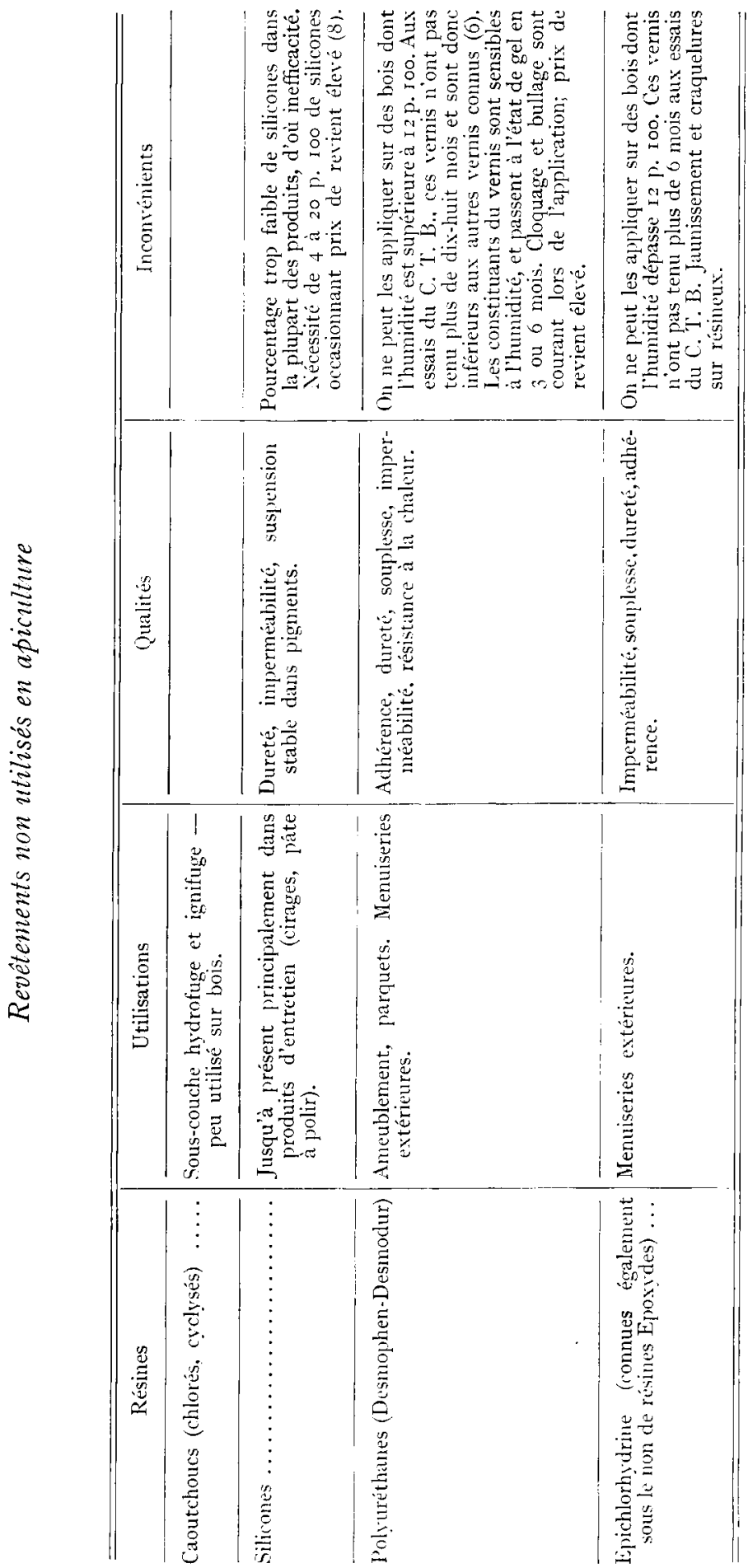

Annales de l'Abrille. - I95\%. 


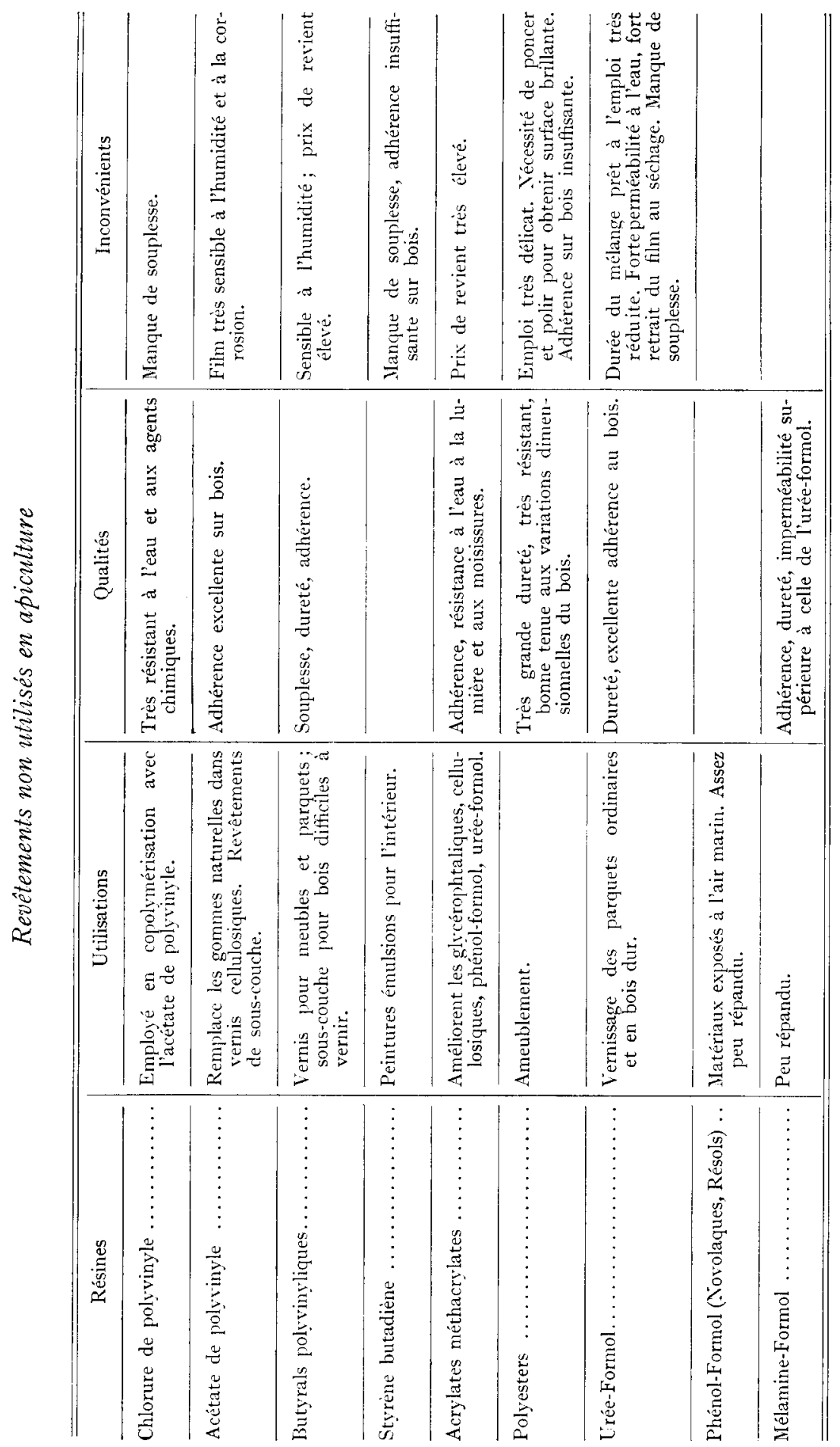


I,es résines artificielles sont des substances macromoléculaires obtenues par synthèse. Nous distinguerons :

Io Les résines polyvinyliques.

$2^{\circ}$ Les résines glycérophtaliques.

$3^{0}$ Les résines polyesters.

$4^{n}$ Les résines polyuréthanes.

$5^{\circ}$ Les résines épichlorhydrine.

60 L es résines urée-Formol.

$7^{\circ}$ Les résines Phénol-Formol.

Ces résines permettent d'obtenir des vernis, préparations qui donnent des films transparents, des peintures dont les films sont opaques et des peinturesímulsions, mélange de deux liquides non miscibles l'un étant dispersé dans l'autre sous forme de fines gouttelettes, qui donnent également des films opacules.

Nous n'envisagerons parmi ces produits que deux catégories susceptibles par leur prix et leur qualités d'être utilisées en apiculture.

Io Copolvmères chloro-acétate de polyoinyle. - Ces résines présentent d'excellentes propriétés telles que résistance à l'eau et aux agents chimiques On y associe de l'acide maléique qui communique une adhérence excellente sur les divers supports. Cette catégorie de revêtements est utilisée sur des bois difficiles à peindre.

Les peintures polyvinyliques actuellement sur le marché ont un prix de revient de $25^{\circ}$ à $35^{\circ}$ francs au mètre carré. I a durée de préservation du bois est de 5 à 6 ans. Elles sont généralement mates.

$2^{\circ}$ Les peintures rlycérophtaliques. - Les résines glycérophtaliques sont également connues sous la dénomination de résines alkydes. Elles proviennent de la polycondensation. de polyacides et de polyols. I,es matières premières les plus utilisées sont l'anhydride phtalique et le glycérol. I eur structure macromoléculaire ne permettant pas leur mise en solution, on les modifie par des huiles siccatives. Il se produit alors un relâchement du résean donnant la possibilité de mettre la résine en solution et fournissant un film plus souple. Pour que le produit puisse sécher à l'air (condition indispensable pour tontes les peintures à bois), le pourcentage d'alkyde pure ne doit pas dépasser $50 \mathrm{p}$. IoO et comporter un siccatif.

Ces peintures présentent le grand avantage d'ètre très souples. Leur adhérence est excellente. La perméabilité à l'eau est faible. P'ar contre la dureté est relativement peu élevée et ces produits résistent mal aux alcalins ; on ne les applique jamais directement sur du ciment, du béton, etc.

Les peintures glycérophtaliques peuvent être appliquées facilement à la brosse, au rouleau et au pistolet, leur délai de séchage est de 12 à 24 heures, on doit passer 3 couches successives pour obtenir une bonne finition et une protection efficace de 5 à 6 ans. Leur prix de revient est plus élevé que celui des peintures à l'huile ordinaires environ $25^{\circ}$ à 350 francs au mètre carré suivant la qualité de la peinture et l'emploi ou non de sous-couche de meilleur marché évitant la première conche de peinture. Une préservation meilleure et une durabilité presque double en font des revêtements nettement supérieurs aux peintures à 1'huile. Cependant les essais en cours à notre Station montrent une très mauvaise tenue de ces peintures, sur pin tout au moins. La présentation sous une forme prête à l'emploi, qui évite des manipulations souvent délicates est un avantage sur les revêtements applicables après adjonction d'un catalyseur. 
Les vernis glycirophtaliques (non pigmentés) présentent les mêmes caractéristiques que les peintures. Un prix de revient à peine inférieur justifie peut leur emploi en apiculture, les revêtements pigmentés étant préférables pour la réverbération des radiations solaires. Ce sont actuellement les vernis les plus sûrs en extérieur.

Le Centre Technique du bois a procédé à des essais de ces vernis pendant plus de deux ans et à ce stade trois vernis glycérophtaliques sur quatre se trouvent en bon état alors qu'un vernis gras sur cinq set1lement s'est bien comporté (5).

Certains utilisateurs appliquent une couche d'impression diluée renfermant un produit insecticide et fongicide destiné à garantit le bois contre l'attaque des insectes et des champignons. Certaines résines alkydes sont employées comme plastifiants dans des revêtements à base de résines artificielles variées.

\section{Produits hydrofuges.}

Ils sont utilisés pour former écran contre l'humidité. En dehors des peintures au caoutchouc chloré, aux résines vinyliques, et des vernis aux silicones précédemment nommés, il existe des produits hydrofuges qui sont apparus en Hurope depuis quelques années. Ils sont appliqués par trempage de quelques minutes ou plus profondément par imprégnation en vase clos (7). La surface du bois ainsi traitée reste propre et peut être peinte. L'application d'un produit hydrofuge avant le revêtement terminal augmente de 50 à roo francs le prix de revient all $\mathrm{m}^{2}$.

\section{Fongicides et insecticides.}

Le bois est à la merci de nombreuses altérations d'origine biologiques. Celles-ci peuvent nuire à la bonne tenue du film de peinture ou provoquer une destruction profonde du bois. Les agents de dégradation sont d'une part les insectes qui occasionnent la vermoulure et les champignons qui entraînent la pourriture du bois sous diverses formes. Des traitements préventifs (appliqués avant montage des objets si possible) sont donc à conseiller, ce sont :

Le carbonyle, constitué par un mélange malheureusement mal défini et peu constant d'huile d'anthracène et d'huile de créosote. Il doit avoir une teinte brun doré et être fluide pour imprégner le bois au nuximum. Certains le rendent visqueux en y ajoutant des goudrons mais alors l'imprégnation obtenue est moins profonde. Les huiles de créosote possèdent d'incontestable: propriétés antiseptiques et le bois imprégné de carbonyle devient imputrescible, mais il prend une teinte foncée pett esthétique. Cependant la teinte s'éclaircit légèrement à longue échéance. Le bois ainsi traité à tendance à se fendre et à se déformer autant, sinon plus, que les bois nus. D'autre part, il est difficile d'appliquer une peinture claire sur des bois imprégnés depuis peu de temps au carbonyle. Les propriétés insecticides du carbonyle sont limitées; il agirait plutôt par son odeur réptulsive. Le prix de revient est extrêmement bas : Io à 30 francs ats $\mathrm{m}^{2}$.

Produits phénoliques : Produits chlorés dérivés de la houille (penta-chlorophénols) qui possèdent d'excellentes propriétés fongicides et quelques propriétés insecticides qui sont améliorées par l'adjonction d'H. C. H. (hexachlorocyclohexane). A noter que 1'H. C. H. est extrêmement toxique pour les abeilles et qu'il convient de s'assurer de l'absence de ce produit dans les insecticides employés sur les ruches. 
Produits naphlaléniques à base de chloronaphtalène. Ces produits chlorés s'emploient an pincean et par trempagc', leur grandc lluidité permet d'inprégner le bois en profondeur. Ils sont compatibles avec les peintures à l'huile (f).

Je récentes recherches ont nontré (pue la créosote, le pentachlorophénol et le naphténate de cuive sont beancony) plus actifs en mélange (que lorsqu'ils sont enployés isolément aux mêmes doses. Il en est cle même lorsque les connbinaisons pentachlorophénol-naphténate de enivre ou créosote naphténate de cuivre sont mélangées à de l'essence.

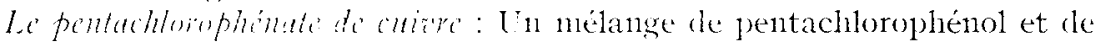
naphténate de cuive provoupe la formation de pentachlorophénate de cuive compose beaucoup moins volatil et moins soluble dans l'eat que le pentachlorophénol. Ce mélange est le plus efficace obtenu jusifu'à présent. Il est en effer 4 fois plus toxicpue comme longicide et insecticide que le pentachlorophénol. Il se présente sous la forme d'un composé brum pourpre assez pen soluble dans les solvants oryaniques. I, pentachlorophenate de cuive peut anssi être formé par la réaction en solution aqueuse, de pentachlorophénate de solium et d'un sel de cuivre soluble dans l'ean comme le sulfate de cuivre.

le pentachlorophénate de cuirre peut aussi être introduit clans le bois par l'internédiaire de solutions aquenses contenant du pentaclulorophénate de sodium et un sel de cuivre mélangé à de l'ammoniarpue ou à un autre produit chimique l'empêchant de réagir sur le pentachlorophénate juscfu'à ce que l'écuilibre soit rompu par l'absorption clans le bois.

I a toxicité des phénols chlorés peut d'autre part ètre considérablement accrue par l'addition en petites quantités d'activants ne possédant pas enxmêmes les propriétés physiques nécessaires leur pernettant de se ranger parmi les produits de préservation de longues durées $(7)$.

l'ous les produits vérifiés par le Centre 'lechniffue du Bois sont excellents, mais malheureusement leurs propriétés insecticides empêchent jusqu'à plus anıle informé leur emploi en apiculture.

Il n'est pas impossible de prévoir néanmoins leur emploi sur les ruches dans certaines conditions que s'attachera à préciser la s'tation lixpérimentale d'Apiculture.

\section{La paraffine.}

I,es paraftines ainsi que les cires microcristallines sont extraites de pétroles bruts paraffiniques. Filles sont constituées d'hỵdrocarbures se présentant à l'état solide sotus forme cristalline. I,es cristaux sont relativement gros, d'un blanc translucide. Iolles sont généralement dutes et cassantes. An contraire, les cires nicrocristallines sont constituées de cristaux plus fins et sont habituellement plastiques.

I,es paraffines sont classées suivant leur teneur en huile et leur point de: fusion en :

Paraffines raffinées. Lorsque la teneur en litule est inférieure à 0,7 p. Ioo. On les distingue entre elles par leur gradlation, c'est-à-dire les limites inférieures: et supérieures entre lesquelles se trouve compris le point de fusion. I,es grarlations extrêmes sont $48^{\circ} / 5^{\circ}$ et $60^{\circ} / 02^{\circ}$.

Paratfines écrilles. Iorsque la teneur en huile est comprise entre $\mathrm{I}, 5$ et 3 p. Ioo. Dewx gradations seulement $50^{\circ} / 5^{\circ}$ et $560^{\circ} / 60^{\circ}$.

Les cires microcristallines sont égalenent classées suivant leur point de fusion. On distingue :

- I es cires microcristallines à haut point de fusion de $80^{\circ}$ à $100^{\circ}$, en géécéral dures et cassintes. 
-... I es cires microcristallines à has point de fusion de $60^{\circ}$ à $60^{\circ}$, flexibles et adhésives.

Les fabricants ont mis au point des mélanges utilisés pour des besoins particuliers pour lesquels les cires ordinaires ne donnaient pas satisfaction.

L es paraffines et cires microcristallines ont trouvé dans l'industrie dess utilisations très variées, en mélanges ou en enrobages. Bougies, cartonnage, caoutchouc, cuir, papiers, produits d'entretien, produits alimentaires, bois.

Le paraffinage des ruches est une méthode de protection asse\% récente: l'esthétique, le luas prix de revient, la rapidité et la facilité d'application sont à la base de l'engouement actuel de certains apiculteurs, pour ce procédé.

L'application se fait par trempage dans un bain de paraffine très chande; les ruches sont ensuite égouttées, puis on laisse refroidir la pellicule de paraffine qui doit être alors mince et lisse (nons présentons dans un chapitre suivant, une analyse du paraffinage montrant les inconvénients qui résultent d'une pratirque trop empirique). On obtient les résultats supérieurs en employant des cires microcristallines an point de fusion avoisinant $80^{\circ}$. Ces cires sont moins sensibles aux variations de température que les paraffines dont le bas point de fusion entraîne parfois sous insolation intense un ramollissement superficiel très désagréable au toucher.

\section{B) Iutres procédés qui pourraient ètre utilisés.}

Les Imprégnations à l'échelle industrielle. - Seules les inprégnations à l'échelle industrielle assurent une pénétration profonde dans le bois (imprégnation par diffusion osmotique et imprégnation en vase clos). Les traverses supportant les rails de chemins de fer sont créosotées par ces procédés. rilles ont une durée d'une trentaine d'années, ce qui est énorme si l'on considère les causes de destruction nécanique, provenant du passage de convois : le contact permanent avec le sol entretient par ailleurs une humidité favorable au développement des champignons.

L'imprégnation par diffusion osmotique. - Ie principe du procédé est basé sur les phénomènes physiques de l'osmose et de la diffusion.

${ }_{\mathrm{I}}^{\mathrm{re}}$ méthode.

Imprégnation des bois verts. . I'tilisée sur les lieux de l'abattage du bois Les troncs écorcés sont badigeonnés avec le produit d'imprégnation. I'antiseptique pénètre lentement par osmose et se diffuse à l'intérieur. Après trois mois l'antiseptique atteint $4 \mathrm{~cm}$ de profonleur. Le phénomène se produit aussi longtemps yu'il y a de la sève dans le bois. Il convient d'éviter les fortes chaleurs qui provoquent un séchage trop rapicle. On utilise cette méthode principalement pour cles poteaux ou bois n'ayant pas à être façonnés après le traitement.

\section{$2^{\text {e }}$ methode.}

Imprégnation des bois humidifiés. - I,e bois est humidifié par trempage. puis l'antiseptique est déposé en surface. si le produit d'imprégnation n'est pas soluble dans l'eau mais dans l'éther, on gonfle le bois avec de l'eau puis on substitue de l'alcool à l'eau, puis de l'éther à l'alcool par trempage dans des bains successifs. Ià, encore, la pénétration profonde est extrêmement lente. Cette méthode est comparable à la préparation des tissus en histologie. 
$3^{\mathrm{e}}$ méthode.

Imprégnation des bois sur pied. - Les méthodes anciennes n'étaient pas très efficaces. L'ingrédient antiseptique étant incorporé à d'autres agents destinés à assurer l'adhérence nécessaire et la pénétration dans le bois se trouvait souvent amoindrie. Une innovation américaine est constituée par une émulsion de pentachlorophénol destinée à traiter le bois sur pied. Ce produit très vis queux peut être facilement étendu sur le bois ; l'épaisseur de la couche à poser dépend de la rétention désirée.

L'émulsion se décompose ensuite graduellement, la surface extérieure se durcit et forme une pellicule de protection sous laquelle se développe une réserve de pentachlorophénol en solution dans l'huile qui est rapidement absorbée par le bois. Un enduit de $\mathrm{I}, 5 \mathrm{~mm}$ aboutit à une plus grande rétention du produit de préservation que l'on peut obtenir avec i5 couches d'une solution huileuse ordinaire du même produit toxique appliquées à la brosse (7).

L'imprégnation en vase clos. - Elle se fait dans de grands autoclaves en forte tôle. Une pression d'une dizaine de kilogrammes permet de faire pénétrer la créosote, ou un autre antiseptique tel que le fluor, très profondément dans le bois. L'injection sous pression assure l'imprégnation la plus régulière et la plus profonde du bois par l'antiseptique.

Elle permet de doser exactement la quantité d'antiseptique incorporée dans le bois. La créosote est efficace contre la totalité des ennemis du bois et son bas prix de revient en facilite l'usage industriel. Sa consistance huileuse imperméabilise le bois et le protège contre le délavage par les eaux de pluie et de l'humidité.

Le hêtre, le pin, le mélèze, peuvent facilement être créosotés profondément. Par contre, le sapin et l'épicéa ne le peuvent qu'après traitement spécial ; les injections d'antiseptique à base de fluor leur sont au contraire facilement appliquées (9).

La créosote noircit le bois et le rend réfractaire aux peintures, sauf à la peinture aluminium. Elle a une odeur persistante et désagréable. Ses exsudations, par forte chaleur, tachent les mains et les vêtements. Ces divers inconvénients rendent impossible l'emploi de la créosote pour la préservation des ruches.

\section{$2^{\text {e PARTIE. }}$}

\section{ETUDE EXPERIMENTALE DU PARAFFINAGE DES BOIS DES RUCHES}

Nons avons indiqué page 246 que le paraffinage des ruches s'obtient dans un bain de paraffine chaude. Dans la pratique le trempage se fait chez l'apiculteur, à l'aide d'un appareillage rudimentaire et mal contrôlé, généralement une simple cuve sur un feu de bois. La température du bain varie alors de $70^{\circ}$ à $\mathrm{I}^{2} \mathrm{O}^{\circ}$ environ. Après égouttage et refroidissement des ruches, il reste sur le bois une pellicule de paraffine d'épaisseur très variable; cette épaisseur dépend de deux facteurs.

Io La température du bain. Plus la paraffine est chaude au moment du trempage, plus la couche adhérant au bois est mince et la pénétration profonde.

$2^{\circ}$ Le temps de refroidissement de la couche de paraffine après le bain. Plus la température ambiante est basse, moins parfait est l'égouttage des ruches. 
De ces deux facteurs mal réglés découle un certain nombre d'inconvénients:

a) Mauvaise imperméabilité du revêtement d'où inefficacité partielle.

b) La couche préservatrice se raye et s'arrache facilement.

c) Les ruches paraissent grasses et désagréables au toucher lorsqu'elles se trouvent au soleil ; les mains glissent sur le bois pendant les manipulations, augmentant ainsi les risques d'accidents.

Nous avons procédé expérimentalement à des essais de paraffinage d'éprouvettes de pin et de sapin. Nos résultats démontrent la nécessité de respecter certaines conditions.

Les éprourettes utilisées ont les dimensions suivantes :

$$
\mathrm{I} 00 \mathrm{~mm}<60 \mathrm{~mm} \therefore 27 \mathrm{~mm}
$$

I a paraffine est de la gradation $5^{6-5} 5^{\circ}$.

Le pin étant beaucoup plus dense (que le sapin (il est en effet presque 50 p. Ioo plus lourd pour la moyenne de nos éprouvettes) nous avons fait des essais similaires, mais séparés, pour ces deux bois, qui sont les plus employés dans la fabrication des ruches.

Les deux premiers tableaux présentent les témoins, c'est-à-dire les épronvettes qui ont été immergées 24 heures dans l'eau sans paraffinage préalable.

\section{'PABLEAU I}

(Eprourettes de sapin).

\begin{tabular}{|c|c|c|c|c|}
\hline $\begin{array}{c}N^{\circ} \text { des } \\
\text { éprouvettes }\end{array}$ & 1 & $\begin{array}{l}\text { l'oids atrant } \\
\text { immersion }\end{array}$ & $\begin{array}{l}\text { Poids aurès immersion } \\
\text { de } 24 \text { h dams l'aul }\end{array}$ & $\begin{array}{c}\text { Pourcentage d'eau absorbée } \\
\text { par l'éprouvette }\end{array}$ \\
\hline & & gr & $g$ & 8 \\
\hline$\theta \mathrm{I}$ & & $5+$ & $7^{\circ}$ & 40,7 \\
\hline 12 & & 55,5 & $7 i$ & $3^{6}, 9$ \\
\hline $1 ;$ & & 56.5 & So & 41,5 \\
\hline If & & 56,5 & 70 & 23,7 \\
\hline 15 & & 55 & 77 & 40 \\
\hline
\end{tabular}

Pourcentage moyen d'eau absorbée pur les éprouvettes : $36,5 \%$.

'TABI,EAU II

(Eprouvettes de pin).

\begin{tabular}{|c|c|c|c|c|c|}
\hline $\begin{array}{l}\text { No des } \\
\text { éprouvettes }\end{array}$ & 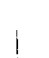 & $\begin{array}{l}\text { Poids avant } \\
\text { inmersion }\end{array}$ & I & $\begin{array}{l}\text { Poids apres immersion } \\
\text { de } 2+\text { h. }\end{array}$ & $\begin{array}{l}\text { Pourcentare d'eau absorbce } \\
\text { par léprouvette. }\end{array}$ \\
\hline$\cdots \quad--\cdots$ & - & -- & & - & 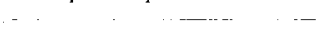 \\
\hline & & $g$ & 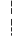 & $g r$ & $\%$ \\
\hline 26 & & $8 \mathrm{r}, 5$ & & 100 & 22,6 \\
\hline 27 & & $8_{1}, 5$ & & 96,5 & I $8,+$ \\
\hline 28 & | & $8_{7,7}$ & & cos & 9,7 \\
\hline 20) & $\vdots$ & 95,5 & & $10,3,5$ & 8,3 \\
\hline 30 & & 84 & & 115 & $.36,9$ \\
\hline
\end{tabular}

Pourcentage moyen d'eat alsorbée par les éprowettes: 19, I "...

Les éprouvettes de pin absorbent l'eau en moindre quantité et de façon j)lus inégale que les éprouvettes de sapin. Cela s'explique par le fait que le pin est beancoup plus résinenx que le sapin, et dans des proportions très variables suivant les zones du tronc d'où proviennent les éprouvettes. 
Nous avons classé nos séries de paraffinage en deux groupes :

- Paraffinage à $120^{\circ}$, dont les résultats sont les plus probants.

-- Paraffinage à $70^{\circ}$.

Io Parafinage ì $\mathbf{2} 20^{\circ}$.

'TABIEAI: III

(Eprouvettes de sapin.)

I minute dans le bain de paraffine.

Immersion 24 heures dans l'eau après 4 heures de séchage.

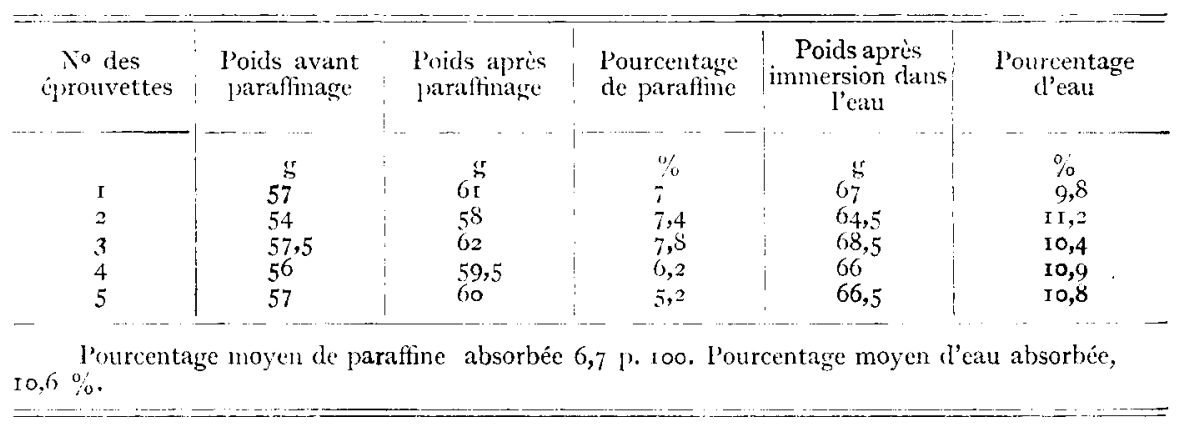

\section{TABI, EaU IV}

(Eprouvettes de Pin).

I minute dans le bain de paraffine.

Inmersion 24 heures dans l'eau après 4 heures de séchage.

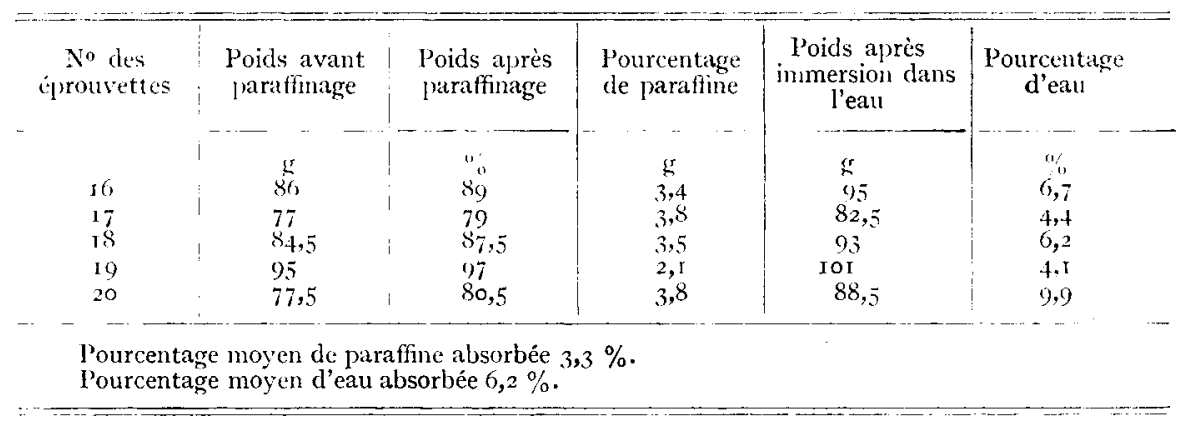

La paraffine, comme l'eau, pénètre en moindre quantité dans les éprourettes de pin que dans les éprouvettes de sapin.

La couche superficielle de paraffine est mince et adhère bien au support.

On note un ralentissement très sensible de la pénétration de l'eau par rapport à la pénétration dans les éprouvettes témoins.

(Ex : Sapin 6,7 p. 100, pour 36,5 p. Ioo dans les témoins).

Le temps de séchage des éprouvettes après paraffinage aurait pu influer sur la pénétration de l'eau. Il n'en est rien. On ne constate pas de différence 
notable dans les pourcentages de pénétration d'eau comne le montrent les tableaux $\mathrm{V}$ et $\mathrm{VI}$.

\title{
'lableau V
}

\section{Eprouvettes de sapin.}

I minute dans le bain de paraffine.

Innmersion 24 heures dans l'eau après I 5 jours de séchage.

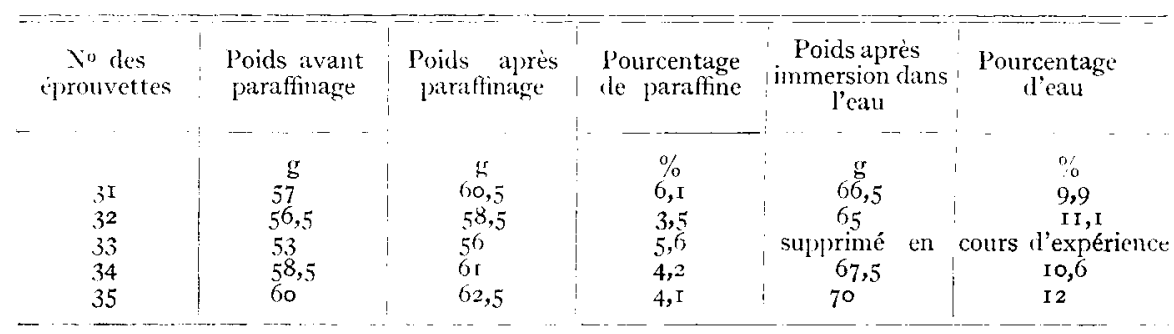

Pourcentage moyen de paraffine absorbée 4,7\% Pourcentage moyen d'eau absorbée 10,9\%

\author{
'TABI,EAU II \\ (Eprouvettes de pin). \\ I minute dans le bain de paraffine.
}

Inmmersion 24 heures dans l'eau après I5 jours de séchage.

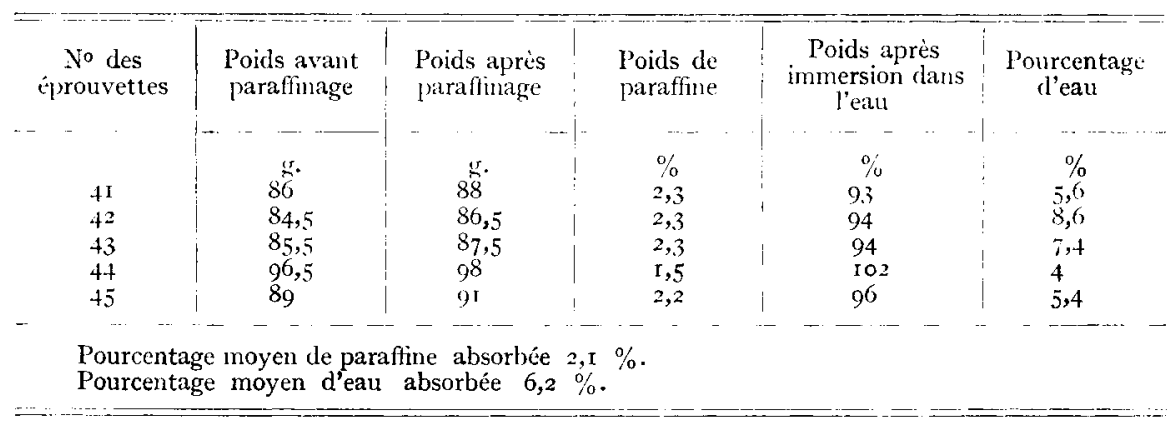

Nous avons ensuite porté le temps de paraffinage à 5 minutes puis à $I_{5}$ minutes afin de découvrir les améliorations possibles de l'hydrofugeage obtenu. Or les moyennes (tableaux VII-VIII-IX et $\mathrm{X}$ ) ne font apparaitre aucune supériorité de ces bains de 5 minutes et 5 minutes sur ceux de I minute.

Par contre si l'hydrofugeage est sensiblement égal, l'aspect extérieur diffère. I, a couche superficielle de paraffine diminue et la pénétration augmente en fonction de l'allongement du temps de paraffinage. Ainsi le poids de paraffine reste presque le même dans tous les cas. 
'TABLEAU VII

Paraffinage d'épronvettes de sapin.

5 mintutes dans le bain de paraffine.

Immersion dans l'eau après $4 \mathrm{~h}$ de séchage.

\begin{tabular}{|c|c|c|c|c|c|}
\hline $\begin{array}{c}N^{\circ} \text { des } \\
\text { éprouvettes }\end{array}$ & $\begin{array}{l}\text { Poids avant } \\
\text { paraffunage }\end{array}$ & $\begin{array}{l}\text { Poids après } \\
\text { paraffinage }\end{array}$ & $\begin{array}{l}\text { Pourcentage } \\
\text { de paraffine }\end{array}$ & $\begin{array}{c}\text { l'oids après } \\
\text { immersion dans } \\
\text { l'eau }\end{array}$ & $\begin{array}{l}\text { Pourcentage } \\
\text { d'eatl }\end{array}$ \\
\hline & $g$ & $\mathrm{~g}$ & $\%$ & 8 & $\%$ \\
\hline $\mathrm{I} 3 \mathrm{I}$ & 57,5 & 60 & 4,3 & 67 & 11,6 \\
\hline 132 & 58 & 60,5 & 4,3 & 68 & I 2,3 \\
\hline 133 & 56 & 58 & 3,5 & 66 & 13,7 \\
\hline 134 & 60,5 & 63,5 & 4,9 & $i^{2}$ & 13,3 \\
\hline 135 & 62 & 64 & 3,2 & 71 & 10,9 \\
\hline
\end{tabular}

Pourcentage noyen de paraffine absorbée $4 \%$.

Pourcentage moyen d'eau absorbée $12,3 \%$.

\section{TABLEAU VIII}

Paraffinage d'éprouvettes de sapin.

I5 minutes dans le bain de paraffine.

Immersion dans l'eau après 4 heures de séchage.

\begin{tabular}{|c|c|c|c|c|c|}
\hline $\begin{array}{c}\text { No des } \\
\text { éprouvettes }\end{array}$ & $\begin{array}{l}\text { Poids avant } \\
\text { paraffinage }\end{array}$ & $\begin{array}{l}\text { Poids après } \\
\text { paraffinage }\end{array}$ & $\begin{array}{c}\text { Pourcentage } \\
\text { paraffine }\end{array}$ & $\begin{array}{l}\text { Poids après } \\
\text { immersion dans } \\
\text { l'cau }\end{array}$ & $\begin{array}{l}\text { Pourcentage } \\
\text { d'eau }\end{array}$ \\
\hline & $\mathrm{g}$ & gr & $\%$ & $\mathrm{gr}$ & $\%$ \\
\hline${ }_{1} 3^{6}$ & 60,5 & 62 & 2,4 & 69 & II, 2 \\
\hline 137 & 56,5 & 58,5 & 3,5 & 66 & I 2,8 \\
\hline 1.38 & 60,5 & 62 & 2,4 & 70 & 12,9 \\
\hline I39 & 57 & 59,5 & 4,3 & 67 & 12,6 \\
\hline I 40 & 63 & 65 & $3, \mathrm{I}$ & 72 & $10, \bar{i}$ \\
\hline $\begin{array}{l}\text { Pourcenta } \\
\text { Pourcenta }\end{array}$ & $\begin{array}{l}\text { moyen de pe } \\
\text { moyen d'eau }\end{array}$ & $\begin{array}{l}\text { fine absorl } \\
\text { osorbée }\end{array}$ & $\begin{array}{r}3, \mathrm{I} \% \\
\mathrm{I} 2 \%\end{array}$ & & \\
\hline
\end{tabular}

\section{TABLEAU IX}

Eprouvettes de pin.

5 minutes dans le bain de paraffine.

Immersion dans l'eau après 4 heures de séchage.

\begin{tabular}{|c|c|c|c|c|c|}
\hline $\begin{array}{l}\mathrm{N}^{\circ} \text { des } \\
\text { éprouvettes }\end{array}$ & $\begin{array}{l}\text { Poids avant } \\
\text { paraffinage }\end{array}$ & $\begin{array}{l}\text { Poids après } \\
\text { paraffinage }\end{array}$ & $\begin{array}{l}\text { Pourcentage } \\
\text { de paraffine }\end{array}$ & $\begin{array}{c}\text { Poids après } \\
\text { immersion dans } \\
\text { l'eau }\end{array}$ & $\begin{array}{c}\text { Pourcentage } \\
\text { d'eas: }\end{array}$ \\
\hline & $\mathrm{g}$ & $g$ & $\%$ & $\mathrm{~g}$ & $\%$ \\
\hline I4 I & 85 & 88 & 3,5 & 102 & I 5,0 \\
\hline I 42 & $8 \mathrm{I}, 5$ & 82,5 & 1,2 & 89 & 7,8 \\
\hline 143 & $8 \mathrm{I}$ & 82 & 1,2 & 89 & 8,5 \\
\hline I 44 & 97,5 & 98,5 & I & 102,5 & 4 \\
\hline 145 & 95,5 & 96,5 & I & 102,5 & 6,2 \\
\hline $\begin{array}{l}\text { Pourcentag } \\
\text { Pourcentag }\end{array}$ & $\begin{array}{l}\text { moyen de pa } \\
\text { moyen d'eau }\end{array}$ & $\begin{array}{l}\text { ine absorbée } \\
\text { sorbée }\end{array}$ & $\begin{array}{l}\mathrm{I}, 5 \% \\
8,4 \% .\end{array}$ & & \\
\hline
\end{tabular}




\section{TABLEAU X}

\section{Eprouvettes de pin.}

I5 minutes dans le bain de paraffine.

Immersion dans l'eau après 4 heures de séchage.

\begin{tabular}{|c|c|c|c|c|c|}
\hline $\begin{array}{c}\text { No des } \\
\text { cprouvettes }\end{array}$ & $\begin{array}{l}\text { Poids avant } \\
\text { paraffinage }\end{array}$ & $\begin{array}{l}\text { Poids après } \\
\text { paraffinage }\end{array}$ & $\begin{array}{l}\text { Pourcentage } \\
\text { de paraffinc }\end{array}$ & $\begin{array}{c}\text { Poids après } \\
\text { immersion dans } \\
\text { l'call }\end{array}$ & $\begin{array}{c}\text { Pourcentage } \\
\text { d'e:ul }\end{array}$ \\
\hline & $\mathrm{g}$ & $\mathrm{gr}$ & $\%$ & rr & 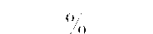 \\
\hline 146 & 85,5 & $89^{5}$ & 4 & $\begin{array}{c}\vdots \\
10.3\end{array}$ & 15,7 \\
\hline$x+7$ & 80 & $8 I, 5$ & 1,8 & 88 & 7.9 \\
\hline 348 & 82 & 83 & $\mathrm{I}, 2$ & 80 & 7.2 \\
\hline 140 & 86,5 & 89 & 2,3 & 99,5 & ri, 7 \\
\hline 150 & 79 & 84 & 5,9 & 15 & 1.3 \\
\hline
\end{tabular}

Pourcentage moyen de paraffine absorbée 3,r \%.

Pourcentage moyen d'eau absorbée Ir, $\mathrm{x} \%$.

Nous avons alors porté la durée du paraffinage à 24 heures, afin de voir si, dans ces conditions extrêmes (et irréalisables dans la pratique) des changements notables intervenaient dans les résultats.

Ėn effet, la proportion de paraffine pénétrant dans les pores des épronvettes est énorme (tableaux XI, XII, XIII, XIV). Le prix de revient d'une telle méthode est excessif tant par la quantité de paraffine utilisée que par le très long temps de chauffe nécessaire. I'ailleurs on n'élimine pas complètement pour autant la pénétration de l'ean dans les épronvettes. La conche de paraffine restant en surface est infime.

\section{TABLEAU XI}

\section{Eprouvettes de sapin.}

24 heures dans le bain de paraffine.

Immersion dans l'eaı après 4 heures de séchage.

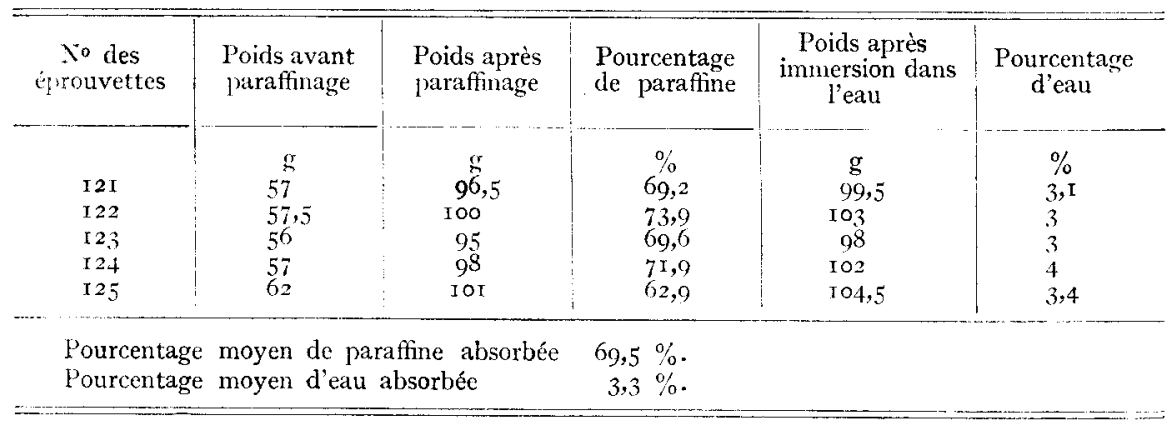




\section{Tambiau XII}

Epronzettes de pin.

24 heures dans le bain de paraffine.

Immersion dans l'eau après 4 heures de séchage.

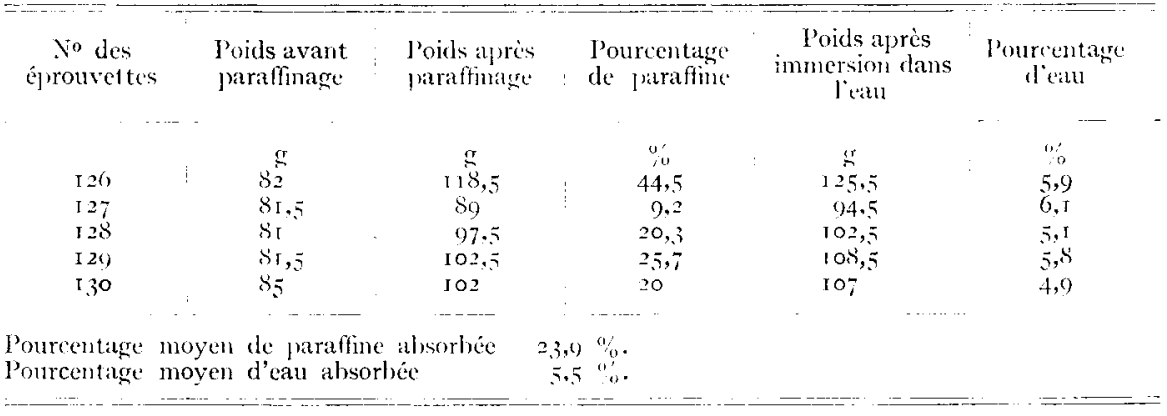

\section{T'ABI,IAU XIII}

Eprouicttes de sapin.

24 heures dans le bain de paraffine.

Immersion dans l'eau après $I_{5}$ jours de séchage.

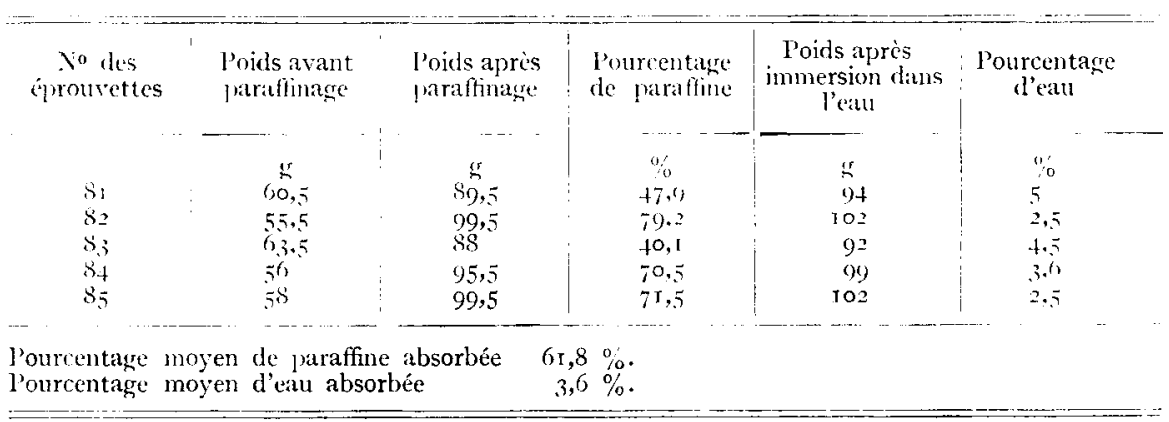

\section{TABI,LAU XIV}

Eprouvettes de pin.

24 heures dans le bain de paraffine.

Immersion dans 1'eau après 15 jours de séchage.

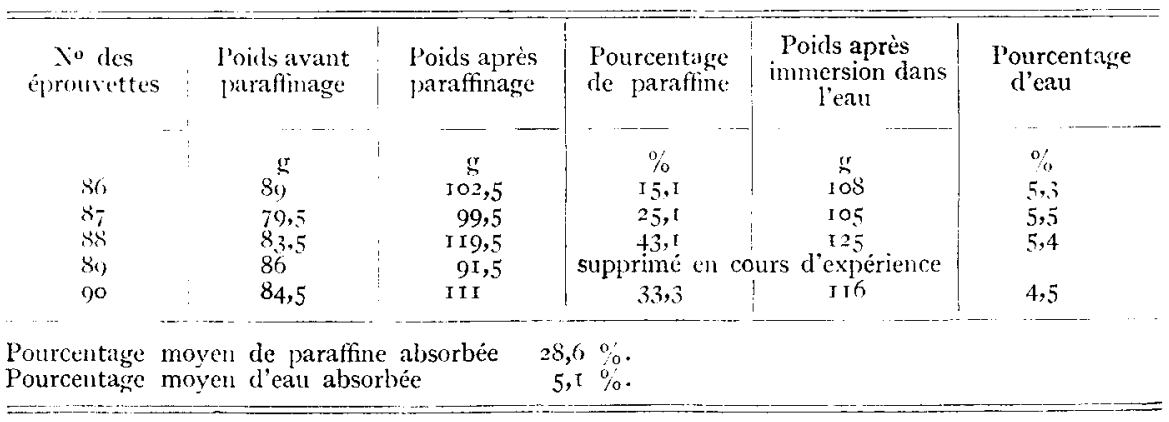


Dans cette série d'expériences la paraffine était toujours maintenue à I $20^{\circ}$.

Il était cependant nécessaire de connaitre les résultats du paraffinage à une température beancoup plus basse. I,es tableaux XV et XVI ont été établis d'après les éprouvettes paraffinées à $70^{\circ}$. Ils montrent que le poids de paraffinte retenue par le bois est comparable à celui de l'expérience à $120^{\circ}$ pour un même temps de trempage, et que l'eau pénètre légèrement plus vite. Mais l'inconvénient majeur de cette méthode apparait dans l'aspect extérieur des éprouvettes. lin effet, sur celles-ci la paraffine est restée en couche épaisse, adhérent très. mal au bois. Elle se boursonfle, se fend et s'arrache facilement.

\section{TABI,EAT: $\mathrm{XY}$ \\ Eprouvettes de pin.}

I minute dans le bain de paraffine à $70^{\circ}$.

Inmersion 24 heures dans l'eau après 4 heures de séchage.

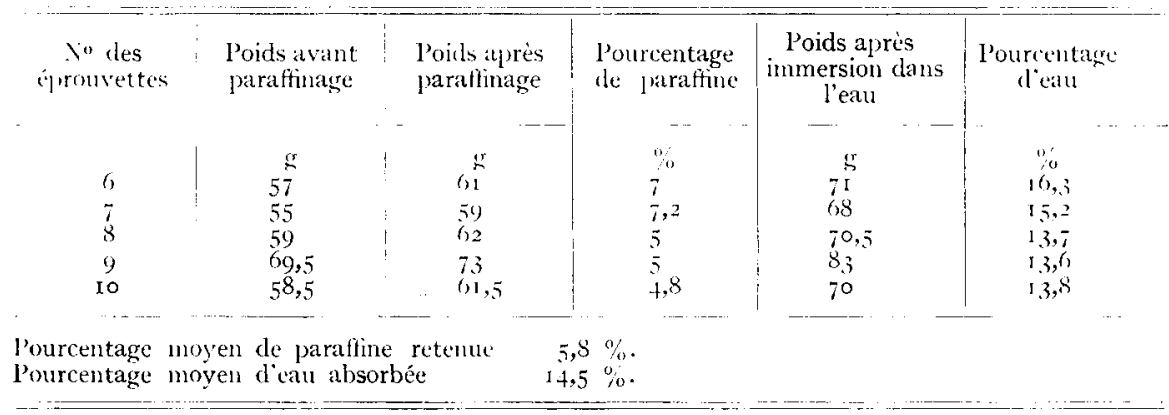

\section{'TABI,FAI XVI \\ Eproutelles de pin.}

I minute dans le bain de paraftine à $70^{\circ}$.

Immersion 24 heures dans l'eau après 4 heures de séchage.

\begin{tabular}{|c|c|c|c|c|c|c|}
\hline $\begin{array}{l}x^{\circ} \text { des } \\
\text { aprouvettes }\end{array}$ & 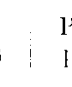 & $\begin{array}{l}\text { l'oids avant } \\
\text { paraflimatgere }\end{array}$ & $\begin{array}{l}\text { Poids atures } \\
\text { paraffinatge }\end{array}$ & $\begin{array}{l}\text { Pourcentage } \\
\text { le jaraffine }\end{array}$ & $\begin{array}{c}\text { Poids a]res } \\
\text { immersion dans } \\
\text { leaul }\end{array}$ & $\begin{array}{l}\text { Pourcentage } \\
\text { d"anu }\end{array}$ \\
\hline \multicolumn{7}{|c|}{-.. } \\
\hline & $i$ & $\ddot{s}$ & ir & u' & $\mathrm{s}$ & $\%$ \\
\hline $2 \mathrm{I}$ & & 78,5 & 82,5 & 5 & $8_{i}$ & 5,4 \\
\hline 22 & & () 1 & 1) 8 & 7,6 & $10 ?$ & 4 \\
\hline 23 & & 85 & i) $\mathrm{T}$ & 7 & 108 & 18,6 \\
\hline 24 & & 81,5 & 87,5 & 7,3 & 100 & 14,2 \\
\hline \multirow[t]{2}{*}{25} & & 32,5 & 88.5 & 7,2 & 92,5 & 4.5 \\
\hline & & -- & 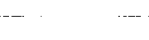 & & $\therefore \quad---$ & \\
\hline l'ourcentage & noyen & en de paraffin & reteme & 0 & & \\
\hline Pourcentage & noyen & en d'cau abso & & $\%$. & & \\
\hline
\end{tabular}

\section{CONCLUSION}

La paraffine, bien que n'étant ni insecticide, ni fongicide, constitue un revêtement valable pour les ruches, principalement comme hydrofuge. Dans: la pratique les ruches ne se trouvent d'ailleurs pas dans des conditions aussi défavorables que dans nos essais. 
Il est cependant indispensable de respecter certaines conditions pour réaliser un paraffinage satisfaisant.

I $^{\circ}$ Le bain de parafine doit être extrêmement chand, Ioo à $120^{\circ}$.

$2^{\circ}$ Le temps de trempage dans le bain doit être assez long a fin de permettre au bois de s'échauffer ce qui donnera une bonne pénétration de la paraffine et surtout un égouttage parfait de la surface du bois.

$3^{\circ}$ I a température ambiante influe également sur cet égouttage en figeant, si elle est trop basse, la pellicule de paraffine au sortir du bain.

En prenant toutes ces précautions, on sera assuré gqu'il ne pénètrera 'fu'un minimum d'eau dans les pores du bois; on obtiendra une surface lisse, sans boursouflure ni fendillement de la paraffine. Nous rappelons que certains apiculteurs possèdent actuellement dans leur ruchers des ruches paraffinées depuis une dizaine d'années et toujours intactes.

\section{CONCLUSION GENERALE}

Les méthodes de préservation des bois en a'uvre à l'extérieur évoluent constamment. Les solutions futures pour la protection des ruches seront certainement fort éloignées des méthodes actuelles. I a plus radicale sera probablement l'abandon du bois au profit des matières plastiques, ou autres produits de synthèse, qui seront insensibles aux agents physiques, chimiques et biologicues.

I)ans l'attente de ces transformations fondamentales

a) L'emploi de fongicides et d'insecticides est particulièrement reconlmandé dans la mesure où ils ne sont pas toxiques pour les abeilles. Ils sont généralement d'un prix de revient peu élevé, et d'emploi facile. J a plupart sont compatibles avec les peintures.

b) I'utilisation d'hydrofuges est également utile en sous-couche. L'huile de lin appliquée à chaud est spécialement recommandée.

c) Les peintures avec des pigments métalliques sont plus clurables que les peintures à l'huile ordinaires.

d) I,es peintures rinyliques possèdent des qualités très jutéressantes, elles s'améliorent d'ailleurs constamment, mais elles sont encore asse\% chères.

e) Le paraffinage des ruches, s'il est exécuté convenablement parait une technique très intéressante, surtout si l'on remplace la paraffine par une cire microcristalline.

\section{RFEIFRENCES BIBLIOGRAPHIQUES}

(I) Laviv: (P.). - L'enregistrement thermique continu dans les populations d'A pis mellifica au cours de l'hivernage. Ins. soc., 1, 39-48, I954.

(2) Büpes, (A.). - Soll eine Bienenwohnung I,uftdurchlässig sein? Arch. Bienenk., 2\%, 29-32, I950.

(3) IABLokov (A. K. H.). - Ethologie de quelques Eilatérides du Mas.sif de Fontainebleau. Mém. Mus. Nat., Hist. Nat. Nour'. Série XVIII, Si r6o, I943.

(4) EBERT (B.). - Peinture et vitrerie. Eyrolles. I953.

(5) Potapov (B.). - Centre 'Technique du Bois. Bul. Inform. Tech., 5, 6-9, I95s.

(6) Girles (G.) et Potapov (B.). - Peinture et vernissage du bois. Cah. Cen. Tech. Bois., 29, 6-13, 195\%. 
(7) Centre Technique du bois. Bull. Inf. Tech., 3, 5, I957.

(8) Rabate (H.). - Que faut-il entendre par l'expression "Peintures aux silicones? "Peint. Pig. Ver. Vol., 33, nº II, 997-999. Edit. Press. Doc. I957.

(9) Peyresaubes (R.). - La préservation du bois. Cah. Cent. Tech. du bois, 5, 2-4, I954. 